\title{
The effect of montelukast in children with asthma predictive index negative episodic viral wheezing
}

\author{
Ayşe Aydoğdu ${ }^{1}$ and Cihan Fidan ${ }^{2}$ \\ ${ }^{1}$ Mersin City Training and Research Hospital \\ ${ }^{2}$ Baskent University Faculty of Medicine
}

June 23, 2021

\begin{abstract}
Background: The effectiveness of montelukast for wheezing in young children is not clear. We aimed to evaluate whether the use of montelukast in the treatment of wheezing affects the number of attacks in children aged 1 to 6 years. Methods: This is a single center study. It was conducted between August 2019 - 2020. Children with aged 0-6 years old with a wheezing complaint who applied to the pediatric allergy and immunology outpatient clinic were included in the study. Modified asthma predictive index of the patients were evaluated. It was classified as episodic viral wheezing (EVW) or multiple trigger wheezing (MTW) based on the type of wheezing. Montelukast treatment was started and the number of attacks was checked. Results: The total of 141 children who applied with wheezing complaints were included in the study. The majority of the patients were boys $(\mathrm{n}=99)$. The mean age of the children was $4.2 \pm 1.5$ years. The median number of attacks before treatment was $2(2-3)$. In $2.8 \%$ of the patients, eczema, in $12.8 \%$ of them, rhinitis and in $26.7 \%$ of them, elevated eosinophils were detected. IgE levels were $148.25 \pm 279.20 \mathrm{IU} / \mathrm{mL}$. According to the number of attacks before treatment, there was a significant decrease in the number of attacks in the control $(p<0.001)$. Conclusion: In this study, montelukast has been shown to reduce the number of EVW attacks and we believe montelukast may be a potential treatment option for EVW in preschool children.
\end{abstract}

\section{INTRODUCTION}

Wheezing is a common public health problem in preschool children, especially in children under one year old, causing frequent hospitalizations. ${ }^{1,2}$ Wheezing is one of the most common respiratory symptoms in childhood, and according to epidemiological studies, the frequency of wheezing is $25 \%$ in the first 3 years and $46 \%$ in the first 5 years. ${ }^{2-5}$ Wheezing child is a heterogeneous situation with different phenotypes. Optimal treatment is required to reduce the frequency and severity of episodes in future period. Since the causes and pathogenesis of wheezing in children are very different, determining the wheezing phenotype and appropriate regulation of the treatment is the most important approach that determines the course of the disease. ${ }^{5-7}$

The simplest clinical classification of wheezing: ${ }^{2,3,5,8}$ episodic viral wheezing (EVW) and multiple trigger wheezing (MTW). The most common phenotype is EVW. Episodic viral wheezing is characterized by the fact that the wheezing attack is triggered by a viral upper respiratory tract infection (URTI) and the patient is asymptomatic between the attacks. In this group, respiratory tract infection is usually evaluated clinically, not by microbiological diagnosis. Among the most causative agents are rhinovirus, respiratory syncytial virus (RSV), coronavirus, human metapneumovirus, parainfluenza virus and adenoviruses. ${ }^{2,5}$ Although episodic viral wheezing usually regresses around the age of 6 , some patients may continue at school age or turn into the MTW phenotype. ${ }^{8}$ In the second type of MTW, attacks can be triggered not only by viral respiratory infections but also by allergens, air pollutants or exercise. ${ }^{8}$

Differentiation of temporary or persistent wheezing in early childhood is an important problem. Since wheezing attacks in the early period of life may be the first sign of asthma that may develop later, determining 
the risk factors for the development of asthma in infancy is important in terms of prognosis. However, there is no biochemical or genetic marker that predicts the development of asthma in small children. Asthma predictive index (API) has been developed to predict who will develop asthma among children under five years age with recurrent wheezing. ${ }^{9,}{ }^{10}$ After the first definition of this index, the criteria were reviewed and the modified asthma predictive index (mAPI) was defined. ${ }^{10}$ If mAPI is negative in the first 3 years, it is predicted that very probably there will be no asthma at 6 years of age, whereas if mAPI is positive in the first 3 years, it is predicted that the possibility of developing asthma in the future is higher. ${ }^{11}$

Leukotriene receptor antagonists (LTRAs) block cysteinyl leukotriene receptors, which are known to be very important in wheezing pathophysiology. Leukotrienes are proinflammatory mediators released mainly by mast cells, triggering bronchoconstriction, eosinophil chemotaxis and mucus secretion in the respiratory tract, causing increased vascular permeability. After all, LTRA's have significant anti-inflammatory and bronchoprotective effects. ${ }^{4,7,12}$ However, the results of studies investigating the effect of the use of LTRAs as maintenance or intermittent therapy on the frequency and severity of attacks in children with EVW are contradictory. ${ }^{13-15}$ The aim of this study is to evaluate the effectiveness of montelukast in pediatric patients with episodic wheezing and API negative.

\section{MATERIAL AND METHODS}

This is a single center study. Patients who applied with recurrent wheezing attacks to the pediatric allergy outpatient clinic at Mersin City Hospital between 01/07/2019 - 01/08/2020 were included in the study. In patients with recurrent wheezing between the ages of $0-6$, physical examinations were performed and eosinophil, total $\operatorname{IgE}$, food mix sps IgE (milk, egg, soy wheat, nuts, fish), phadiotop (inhalant allergen sps IgE), skin prick tests were performed and the results were recorded in their files. The skin prick test was conducted with dermatophagoides pteronisinus, dermatophagoides farinae, alternaria, grain pollen, grass pollen, weed pollen, cat dog epithelium.

Episodic viral wheezing was evaluated as wheezing that develops following a URTI and is characterized by episodes without complaints between wheezing episodes. Upper respiratory tract infection was defined as the persistence of at least 2 of the following 5 symptoms: sneezing, coughing, nasal congestion, runny nose or fever $\left(38.0^{\circ} \mathrm{C}\right)$ for 2 consecutive days.

The mAPI of all patients was evaluated. According to this index, at children who had four or more wheezing episodes, having 1 major or 2 minor criteria was considered positive.

Modified asthma predictive index:

Major criteria: 1. Doctor-diagnosed asthma in mother or father 2. Doctor-diagnosed atopic dermatitis 3. Sensitivity to at least one aeroallergen and Minor criteria: 1. Food allergy 2. Wheezing other than URTI 3. It was evaluated as eosinophilia ([?]4\%) in peripheral blood (Table 1). ${ }^{16}$

Table 1. mAPI

1. A history of [?]4 wheezing episodes with [?]1 physician's diagnosis.

2. In addition, the child must meet [?]1 of the following major criteria or [?]2 of the following minor criteria:

mAPI: modified asthma predictive index

Montelukast therapy to patients was started as $4 \mathrm{mg} /$ day under 5 years of age, $5 \mathrm{mg} /$ day for those 5 years and they were asked to take it regularly every day. The first control was done in the second month and the second control in the fifth month. During the control, the patients were questioned whether they had an attack of wheezing, their physical examinations were performed, and the response to montelukast was evaluated. Montelukast was generally well tolerated and was discontinued in only 1 patient because of discomfort and was excluded from the study. No significant side effects were reported in other patients. 
This study was in accordance with the ethical standards of the institutional and/or national research committee and with the 1964 Helsinki declaration and its later amendments or comparable ethical standards. This research project is approved by The Research and Ethics Committee of Mersin University with the project number 2020/790.

\section{Statistical Analysis}

Mean Standard Deviation, Median, Minimum, Maximum values were given in descriptive statistics for continuous variables, and percentages were given for discrete variables. The compliance of the variables to normal distribution was examined using the Shapiro-Wilk test. Wilcoxon test was used to compare the pre-treatment and 1st control attack numbers of the patients. Mann Whitney U test was used to compare the number of attacks between groups. Chi-Square and Fisher's Exact tests were used for group comparisons of nominal variables. IBM SPSS Statistics version 21 program was used for evaluations and $\mathrm{p}<0.05$ was accepted as the statistical significance limit.

\section{RESULTS}

The total of 141 children who applied with wheezing complaints were included in the study. $29.8 \%$ of the patients were girls $(\mathrm{n}=42)$ and $70.2 \%$ were boys $(\mathrm{n}=99)$. The mean age of the children was $50.18 \pm 17.66$ (15-76) months. The mean age of first attack was $12.73 \pm 5.53$ months, and the median number of attacks before treatment was 2 (2-3). In $2.8 \%$ of the patients, eczema, in $12.8 \%$ of them, rhinitis and in $26.7 \%$ of them, elevated eosinophils were detected. IgE levels were $148.25 \pm 279.20 \mathrm{IU} / \mathrm{mL} .3 .3 \%$ of the patients had Fx5 food allergy, $12.6 \%$ had a Phdiotop inhaler screening panel, and $25.9 \%$ had a positive prick test. API indexes were positive in $28.4 \%$ of patients and $84.4 \%$ of them had EVW.

There were 141 patients who had their first controls and 95 patients who had their second controls. When the number of attacks before treatment and control attacks were compared, a difference was found between the patients $(\mathrm{p}<0.001)$. The decrease in the number of attacks was found to be statistically significant (Table 2).

Table 2: The number of before treatment and control attacks of the patients

\begin{tabular}{lll}
\hline $\mathrm{n}=95$ & Median (Min-Max) & $\mathrm{p}$ \\
\hline Number of attacks before treatment & $2(2-3)$ & $<0.001$ \\
1st control number of attacks & $0(0-1)$ & \\
2nd control number of attacks & $0(0-1)$ & \\
\hline
\end{tabular}

According to the number of attacks before treatment, there was a significant decrease in the number of attacks in the 1st control and 2nd control (respectively; $\mathrm{p}<0.001, \mathrm{p}<0.001$ ). There was no difference between the number of attacks in the 1st control and 2nd control $(\mathrm{p}>0.05)$. Therefore, the subsequent evaluations were made on the patients who received the first control.

There was a difference between the number of attacks before treatment and the number of 1st control attacks in patients with negative modified API $(\mathrm{p}<0.001)$. The number of attacks in the 1st control was significantly lower than before treatment (Table 3 ).

Table 3: The number of before treatment and 1st control attacks in patients with negative and positive mAPI scores

\begin{tabular}{llll}
\hline & mAPI negative & mAPI pozitive & $\mathrm{p}$ \\
\hline & Median (Min-Max) & Median (Min-Max) & \\
Number of attacks before treatment & $2(2-3)$ & $2.5(2-3)$ & 0.491 \\
1st control number of attacks & $0(0-2)$ & $2(0-2)$ & $<0.001$ \\
\hline
\end{tabular}


mAPI: modified asthma predictive index

There was a difference between the number of decreases in the number of attacks of the patients with negative and positive mAPI scores in the 1st control compared to the pre-treatment $(\mathrm{p}<0.001)$. In patients with negative mAPI score, the decrease in the number of attacks in the 1st control was greater than those with positive mAPI scores compared to the pre-treatment. That is, those with negative mAPI scores appear to have a greater decrease in the number of attacks.

A difference was determined between the number of attacks before treatment and the number of 1st control attacks in patients with EVW and MTW. The number of attacks in the 1st control was significantly lower than before treatment (Table 3). There was no difference between the pre-treatment attack numbers of the patients with EVW and MTW ( $>>0.05$ ). A difference was determined between the number of attacks in the 1st control of the patients with EVW and MTW ( $<<0.001)$. Patients with MTW had significantly more attacks in the 1st control than patients with a median of EVW (Table 4).

Table 4: Difference between before treatment and 1st control episodes in patients with EVW and MTW

\begin{tabular}{llll}
\hline & EVW & MTW & p \\
\hline & Median (Min-Max) & Median (Min-Max) & \\
Number of attacks before treatment & $2(2-3)$ & $2(2-3)$ & 0.995 \\
1st control number of attacks & $0(0-2)$ & $2(0-2)$ & 0.000 \\
\hline
\end{tabular}

EVW: episodic viral wheezing; MTW: multiple trigger wheezing

No difference was determined between the gender distribution of the patients with negative and positive mAPI scores $(\mathrm{p}>0.05)$. A difference was determined between the ages (months) and $\operatorname{IgE}$ values of the patients with negative and positive mAPI scores $(\mathrm{p}<0.01)$. Patients with positive mAPI scores were older than those with negative mAPI scores. A difference was determined in the rates of eczema, rhinitis, eosinophilia, fx5 food allergy, phdiotop inhaler allergy and prick skin test positivity in patients with negative and positive mAPI scores $(\mathrm{p}<0.01)$, and these were higher in patients with positive mAPI scores. A difference was determined between the rates of EVW and MTW in patients with negative and positive mAPI scores $(\mathrm{p}<$ 0.001). Modified API was determined positive in $16.8 \%$ of children with EVW, while $90.9 \%$ of those with MTW were determined to be positive.

\section{DISCUSSION}

Episodic viral wheezing attacks are common in preschool children and are associated with morbidity. ${ }^{1,2,6}$ Therefore, it has been the subject of many studies. In this study, the effect of montelukast on the frequency of wheezing in children with mAPI negative EVW was investigated. Daily use of montelukast has been shown to significantly reduce wheezing episodes in patients with EVW who are mAPI negative.

Episodic viral wheezing differs from MTW in that it follows viral URTI and patients are completely free of complaints except for wheezing. Comparing both groups, children with MTW are more likely to develop asthma. $2,3,8$ When responses to montelukast treatment are added, it will be useful to differentiate and follow-up patients in the clinic in terms of both drug selection and prognosis.

The cysteinyl leukotrienes (CysLTs) are one of the mediators involved in the early stage of allergic reactions, and increase during URTI, which is the main trigger for acute wheezing attacks in children. Also, CysLT1 antagonists inhibit a mediator involved in the early stage of the allergic inflammatory pathway. ${ }^{4,7,17}$ There are many studies in the literature evaluating the use of continuous or intermittent LTRA in the treatment of wheezing in children compared to placebo. ${ }^{12,18-22}$ In these studies carried out until present, the effect of montelukast in the treatment of wheezing has been reported as effective in some studies and not effective in others. ${ }^{13-15}$ Montelukast has been shown to have positive effects on asthma symptoms in the study of Barbara Knorr et al. ${ }^{23}$ In the study of Bisgaard et al. in year 2005, it was found that maintenance 
montelukast treatment decreased the rate of exacerbation compared to placebo. It has been shown to be effective in controlling wheezing attacks triggered by viral infections, especially in children aged 2-5 years. 12 In the study of Robertson et al, children aged 2 to 14 years were evaluated and a moderate effect of montelukast on symptoms was reported. ${ }^{21}$ Bacharier et al. also found that intermittent LTRA reduced severity of symptoms. ${ }^{18}$ In the study conducted by Valovirta et al in 2011, more than half of their patients had API positivity, and it was stated that maintenance montelukast did not reduce the attacks but the symptom severity. ${ }^{22}$ However, it is thought that the children included in the study were truly undiagnosed and MTW and atopic asthmatics were misclassified as EVW. ${ }^{19}$ In 2014, Nwokoro et al determined a small but statistically significant reduction in medical care rates in children with the EVW phenotype. ${ }^{20}$

The reason for these different results is the complex and heterogeneous structure of different endotypes in children with wheezing. ${ }^{7}$ Also, there is inconsistency as to whether EVW should be considered a separate entity from MTW, which has an asthma-like phenotype. As a result, the inclusion criteria of patients differ between studies. ${ }^{14}$ In some of these studies, the age of the patients was not limited to under 5 years of age, or the API was not differentiated, and it is likely that children with a distinct EVW phenotype were not a homogeneous group in the studies. ${ }^{4}$ These may inadvertently cause bias in studies, and it should be noted that the rate of unresponsiveness of some patient subgroups to LTRA therapy may vary, as the exact pathophysiology was not determined.

In our study, we classified children under the age of 6 according to mAPI, followed them as EVW and MTW, and evaluated their responses to montelukast use. Studies have shown that most patients improved during follow-up. ${ }^{3,12,18}$ In our study, we wanted to show that montelukast can be much more effective at mAPI negative EVW. While before montelukast, wheezing followed each URTI complaint, they stated that they recovered only with upper respiratory tract symptoms without wheezing after montelukast. This suggested that montelukast may have limited the viral disease picture. In recent studies conducted with the emergence of Covid infection; montelukast has been shown to inhibit irreversible viral infection by acting on the viral genome. ${ }^{24}$ In an insilico study, it has been shown that montelukast has anti-viral activity by interfering with the entry of the virus into the host cell. ${ }^{25}$ Razi et al. study shows that the immunostimulant given in atopy-negative children is effective. This suggests that EVW under 5 years old may be an immune defect that is insufficient to limit viral infection. ${ }^{26}$ In fact, it should not be generalized as asthma under the age of 5 , but should be considered as 2 different entities. However, it seems that more studies are needed on this subject. The most important limitation of our study is the small sample size and the lack of long-term results of the patients. It was also a single center study.

\section{CONCLUSION}

Wheezing occurs under the age of 5 for a variety of reasons. If the patient with recurrent wheezing is evaluated well from the first admission and treated according to wheezing after underlying diseases such as gastroesophageal reflux, cystic fibrosis, ciliary dyskinesia, cardiac and pulmonary anomalies are ruled out, both hospital admissions and attacks will be prevented due to unnecessary treatments and exacerbations of wheezing. In this study, it was observed that the frequency of exacerbation was reduced by $90.9 \%$ in the first control with the use of montelukast in the group with mAPI negative EVW. It was seen that this effect continued in the 2 nd control. With this study, we believe montelukast may be a potential treatment option for EVW in preschool children. This approach is an attractive therapeutic option since it has the potential to reduce systemic steroid exposure and hospitalizations. Studies with a larger patient population are needed to better elucidate the role of montelukast in the management of EVW.

\section{REFERENCES}

1. Bacharier LB. Viral-induced wheezing episodes in preschool children: approaches to therapy. Curr Opin Pulm Med 2010; 16: 31-5.

2. Bloomberg GR. Recurrent wheezing illness in preschool-aged children: assessment and management in primary care practice. Postgrad Med 2009; 121: 48-55. 
3. Brand PL, Baraldi E, Bisgaard H, Boner AL, Castro-Rodriguez JA, Custovic A, et al. Definition, assessment and treatment of wheezing disorders in preschool children: an evidence-based approach. Eur Respir J 2008; 32: 1096-110.

4. Brodlie M, Gupta A, Rodriguez-Martinez CE, Castro-Rodriguez JA, Ducharme FM and McKean MC. Leukotriene receptor antagonists as maintenance or intermittent treatment in pre-school children with episodic viral wheeze. Paediatr Respir Rev 2016; 17: 57-9.

5. Bhatt JM and Smyth AR. The management of pre-school wheeze. Paediatr Respir Rev 2011; 12: 70-7.

6. Ducharme FM, Tse SM and Chauhan B. Diagnosis, management, and prognosis of preschool wheeze. Lancet 2014; 383: 1593-604.

7. Haq I, Harris C, Taylor J, McKean MC and Brodlie M. Should we use montelukast in wheezy children? Arch Dis Child 2017; 102: 997-8.

8. Schultz A and Brand PL. Episodic viral wheeze and multiple trigger wheeze in preschool children: a useful distinction for clinicians? Paediatr Respir Rev 2011; 12: 160-4.

9. Castro-Rodríguez JA, Holberg CJ, Wright AL and Martinez FD. A clinical index to define risk of asthma in young children with recurrent wheezing. Am J Respir Crit Care Med 2000; 162: 1403-6.

10. Expert Panel Report 3 (EPR-3): Guidelines for the Diagnosis and Management of Asthma-Summary Report 2007. J Allergy Clin Immunol 2007; 120: S94-138.

11. Castro-Rodriguez JA. The Asthma Predictive Index: early diagnosis of asthma. Curr Opin Allergy Clin Immunol 2011; 11: 157-61.

12. Bisgaard H, Zielen S, Garcia-Garcia ML, Johnston SL, Gilles L, Menten J, et al. Montelukast reduces asthma exacerbations in 2- to 5-year-old children with intermittent asthma. Am J Respir Crit Care Med 2005; 171: 315-22.

13. Burman A. Question 2: Is there a role for Montelukast in the management of viral-induced wheeze in preschool children? Arch Dis Child 2018; 103: 519-20.

14. Hussein HR, Gupta A, Broughton S, Ruiz G, Brathwaite N and Bossley CJ. A meta-analysis of montelukast for recurrent wheeze in preschool children. Eur J Pediatr 2017; 176: 963-9.

15. Kaiser SV, Huynh T, Bacharier LB, Rosenthal JL, Bakel LA, Parkin PC, et al. Preventing Exacerbations in Preschoolers With Recurrent Wheeze: A Meta-analysis. Pediatrics 2016; 137.

16. Guilbert TW, Morgan WJ, Zeiger RS, Bacharier LB, Boehmer SJ, Krawiec M, et al. Atopic characteristics of children with recurrent wheezing at high risk for the development of childhood asthma. J Allergy Clin Immunol 2004; 114: 1282-7.

17. Hansen I, Klimek L, Mösges R and Hörmann K. Mediators of inflammation in the early and the late phase of allergic rhinitis. Curr Opin Allergy Clin Immunol 2004; 4: 159-63.

18. Bacharier LB, Phillips BR, Zeiger RS, Szefler SJ, Martinez FD, Lemanske RF, Jr., et al. Episodic use of an inhaled corticosteroid or leukotriene receptor antagonist in preschool children with moderate-to-severe intermittent wheezing. J Allergy Clin Immunol 2008; 122: 1127-35.e8.

19. Brodlie M, Gupta A, Rodriguez-Martinez CE, Castro-Rodriguez JA, Ducharme FM and McKean MC. Leukotriene receptor antagonists as maintenance and intermittent therapy for episodic viral wheeze in children. Cochrane Database Syst Rev 2015; 2015: CD008202.

20. Nwokoro C, Pandya H, Turner S, Eldridge S, Griffiths CJ, Vulliamy T, et al. Intermittent montelukast in children aged 10 months to 5 years with wheeze (WAIT trial): a multicentre, randomised, placebo-controlled trial. Lancet Respir Med 2014; 2: 796-803. 
21. Robertson CF, Price D, Henry R, Mellis C, Glasgow N, Fitzgerald D, et al. Short-course montelukast for intermittent asthma in children: a randomized controlled trial. Am J Respir Crit Care Med 2007; 175: $323-9$.

22. Valovirta E, Boza ML, Robertson CF, Verbruggen N, Smugar SS, Nelsen LM, et al. Intermittent or daily montelukast versus placebo for episodic asthma in children. Ann Allergy Asthma Immunol 2011; 106: 518-26.

23. Knorr B, Franchi LM, Bisgaard H, Vermeulen JH, LeSouef P, Santanello N, et al. Montelukast, a leukotriene receptor antagonist, for the treatment of persistent asthma in children aged 2 to 5 years. Pediatrics 2001; 108: E48.

24. Aigner L, Pietrantonio F, Bessa de Sousa DM, Michael J, Schuster D, Reitsamer HA, et al. The Leukotriene Receptor Antagonist Montelukast as a Potential COVID-19 Therapeutic. Front Mol Biosci 2020; 7: 610132 .

25. Durdagi S, Avsar T, Orhan MD, Serhatli M, Balcioglu BK, Ozturk HU, et al. The neutralization effect of Montelukast on SARS-CoV-2 is shown by multiscale in silico simulations and combined in vitro studies. bioRxiv 2020: 2020.12.26.424423.

26. Razi CH, Harmancı K, Abacı A, Özdemir O, Hılı S, Renda R, et al. The immunostimulant OM-85 BV prevents wheezing attacks in preschool children. J Allergy Clin Immunol 2010; 126: 763-9.

\section{Hosted file}

Tables.docx available at https://authorea.com/users/421472/articles/527494-the-effectof-montelukast-in-children-with-asthma-predictive-\%C4\%B1ndex-negative-episodic-viralwheezing 